\title{
ALGUNAS CARACTERÍSTICAS ASOCIADAS A LA SATISFACCIÓN DE LAS MADRES DE LOS RECIÉN NACIDOS USUARIOS DEL CONSULTORIO EXTERNO DE NEONATOLOGÍA DEL HOSPITAL III YANAHUARA FEBRERO 2017
}

\author{
Some Characteristics That Are Associated To The Level Of Satisfaction Of External Users Of The \\ Yanahuara's Hospital Outpatient Clinic Of The In February, 2017.
}

Karen Leticia LLerena Ururi ${ }^{1, a}$

1 Puesto de Salud de Chaparra. Caraveli, Arequipa, Perú

a. Médico Cirujano

\section{RESUMEN}

Objetivo: Identificar algunas características asociadas a la satisfacción de las madres de los usuarios del consultorio externo de neonatología de un hospital de la seguridad social peruana nivel III ESSALUD. Material y Métodos: Estudio de asociación, transversal, observacional, prospectivo. Se seleccionó por conveniencia 102 madres de recién nacidos que acuden a los consultorios externos del Hospital III Yanahuara en el mes de febrero 2017. Empleándose la encuesta SERVQUAL modificada. Los datos fueron analizados mediante el programa estadístico SPSS y Excel. Resultados: La edad materna se encontró entre los 18 a 38años, ( $\mathrm{x}=27$ años, $\mathrm{S}=5,89)$. Se halló una satisfacción del usuario del $41,18 \%$ donde las dimensiones más valoradas fueron seguridad $(22,5 \%)$, empatía $(21,6 \%)$, fiabilidad $(14,7)$ capacidad de respuesta $(8,8 \%)$, fiabilidad y la dimensión con mayor insatisfacción fue la de aspectos tangibles $(5,9 \%)$, las características asociadas a la satisfacción fue el ser casada $(p=0.020)$. y el ser primeriza $(p=0.028)$ resultó asociado a menor ocurrencia de satisfacción ( OR: 0,353; IC: $0,140-0,82$ ). Conclusiones: Existe una satisfacción del $41,18 \%$ del usuario externo con la atención médica recibida en los consultorios externos del Hospital III Yanahuara. La satisfacción según dimensiones fue: seguridad (22,5\%), empatía $(21,6 \%)$, fiabilidad $(14,7)$ capacidad de respuesta $(8,8 \%)$, la dimensión con mayor insatisfacción fue la de aspectos tangibles $(5,9 \%)$. Entre las características que se asociaron con la satisfacción de las madres tenemos el estar casada y, el ser tener solo un hijo está asociado a una menor ocurrencia de percepción de satisfacción.

Palabras clave: Satisfacción, calidad de atención, recién nacido sano.

\section{SUMMARY}

Objective: Identify some characteristics that are associated to the level of satisfaction of external users of the outpatient clinic of the III Yanahuara.

Material and Methods: Asociative, transversal, observational, prospective study. Was selected a convenience sample of 102 users of the outpatient clinic of the III Yanahuara Hospital was selected in the month of February 2017.We have used the modified SERVQUAL survey. Data was analyzed analyzed using SPSS statistical software and Excel. Results: There was a satisfaction of the $41.18 \%$ in the user where the dimensions most valued were security $(22.5 \%)$, empathy $(21.6 \%)$, responsive $(78.8 \%)$, reliability (14.7), and the dimension found with more dissatisfaction was found was tangible aspects (5.9\%) . the socio-demographic characteristics associated with the external user insatisfaction was have just one child $(p=0.028)$ and being single $(p=0.020)$. Conclusions: There is a level of external user satisfaction with medical attention of $41.18 \%$, in the III Yanahuara Hospital. It was found that the characteristics associated with the external user satisfaction was being married, and have just one child has been associated to a minor occurrence of satisfaction.

Keywords: Patient satisfaction, quality of attention, healthy new born. 


\section{INTRODUCCIÓN:}

En el proceso de mejora en las organizaciones prestadoras de salud, el compromiso personal con la calidad, no solo es encomiable, sino un paso indispensable en la meta hacia una cultura de calidad. Las experiencias de pacientes, evaluadas mediante encuestas de satisfacción, que actúan como mecanismo asegurador de que las vivencias y opiniones de los pacientes son escuchadas, constituyen elementos útiles para mejorar la calidad de la atención (1). El interés por conocer la satisfacción de los padres en el control del recién nacido en los primeros días de vida ha sido de aparición tardía, y concretamente en nuestro país es un campo aún muy poco explorado. Además que el uso de encuestas para medir satisfacción en este ámbito no está muy difundido (2). Para mejorar la calidad de vida es necesario hacer notar el papel que desempeña la familia, además, contemplar al neonato como parte integrante de la familia y de la sociedad, lo cual ha dado lugar al concepto de los cuidados enfocados en la familia, cada vez más arraigado en los servicios Neonatales de todo el mundo (3). Por otro lado diversas investigaciones demuestran que los usuarios de los servicios de salud que encuentran satisfechos con el servicio también a cumplir con las indicaciones y recomendaciones, esto produce mejores resultados clínicos, de igual manera, es menos probable que los pacientes insatisfechos cumplan las indicaciones, y favorezcan la recomendación del servicio que brinda la Institución (4).

\section{MATERIAL Y METODOS}

El presente estudio es de asociación, observacional, corte transversal, prospectivo, según Altman. La población está formada por todas las madres de los recién nacidos que acuden al consultorio externo de Neonatología del Hospital III Yanahuara EsSALUD en el departamento de Arequipa, Perú, en el periodo de febrero 2017, para su primer control. Se entrevistó a 141 madres. De las cuales, 39 se retiraron antes de terminar la entrevista de las preguntas de la hoja de percepciones, quedando la muestra constituida por 102 madres que respondieron a todas las preguntas de los instrumentos de recolección de datos. Tipo de Muestreo: Causal o accidental.

Criterios De Inclusión: Madres de los recién nacidos que acuden al consultorio de neonatología en el Hospital III Yanahuara EsSALUD que hablan o entienden el idioma español; de cualquier edad; que acuden al consultorio externo de neonatología para su primer control.en el Hospital III Yanahuara EsSALUD y que deseen participar del estudio.

Criterios De Exclusión: Madres de los recién nacidos que acuden al consultorio de neonatología en el Hospital III Yanahuara EsSALUD menores de 18 años que no se encuentren acompañadas de alguno o ambos padres o tutor legal; cuyos recién nacidos hayan tenido hospitalización previa y /o tengan alguna patología diagnosticada previamente.

Para la aplicación del instrumento, se solicitaron los permisos correspondientes; se pidió el consentimiento informado en forma verbal a las usuarias. Para la recolección de datos se utilizó la entrevista personal ( 15 a 20 minutos). Se realizó un análisis descriptivo de las variables en el paquete estadístico SPSS 20,0 . Las variables cuantitativas se describen mediante media, mediana, desviación estándar (DE) y rango, y las cualitativas, como proporciones tanto en la satisfacción global y de cada una de las dimensiones de la calidad, datos que fueron expresados en tablas y una gráfica. Se aplicó la prueba de Chi cuadrado y, un análisis regresión logística binaria para la identificación de los factores asociados a la satisfacción. Se trabajó con un nivel de significancia estadística del $5 \%$.

LA ENCUESTA SERVQUAL: Ha sido específicamente diseñada para el ámbito hospitalario público de países de habla hispana y que reúne la ventaja de un número 
reducido de ítems, 22 en total, que abarca los elementos básicos en la evaluación de la calidad percibida, y a diferencia de otras propuestas, combina expectativas $y$ percepciones para obtener una medida compatible con el "paradigma de la desconfirmación" (5). Esta nueva escala de respuesta resulta mucho más sencilla para el paciente, supera el inconveniente de preguntar por las expectativas una vez recibida la atención y es compatible con estudios (cuantitativos o cualitativos) para determinar las expectativas de los pacientes 0 de sus familiares. Fue validado en el 2012 por investigadores de la (UPCH) Universidad Privada Cayetano Heredia (6). Para la calificación de las expectativas y percepción se utilizó una escala numérica del 1 al 7 , considerando 1 la más baja y 7 , la más alta. Como se hizo en el trabajo de CasalinoCarpio (7), se consideró: el grado de satisfacción de los usuarios calculando la diferencia entre las respuestas brindadas para las expectativas y las percepciones; posteriormente se interpretó según los siguientes parámetros: menor 0 igual a $0=$ satisfacción; mayor que 0 = insatisfacción.

\section{RESULTADOS}

En las características sociodemográficas de la población de estudio se encontró: de las 102 madres entrevistadas en el Hospital III Yanahuara el grupo etario que predomina se encuentra entre los rangos de 21 a 35 años $(79,4 \%)$, en cuanto al nivel socioeconómico los resultados indican que la mayoría tienen un nivel socioeconómico medio $(57,8 \%)$ medido a través de la Escala Socioeconómica APEIM. La mayoría de madres entrevistadas tienen un grado de instrucción secundaria (52\%). No se halló ninguna madre analfabeta o con instrucción primaria. Con relación al tipo de parto un $70 \%$ de las madres han dado a luz por parto normal y un $30 \%$ por cesárea. Se aprecia que existe una insatisfacción global en un $58,8 \%$, lo que quiere decir que la madre percibe la atención menos de lo que esperaba. (Tabla 1). Se observó que la satisfacción global de las madres del estudio se encuentra en el estándar menor de $60 \%$ $(41,18 \%)$. Respecto al nivel de satisfacción el $68,27 \%$ de las insatisfechas corresponde a las madres solteras. Podemos afirmar que existe asociación estadística, de la satisfacción y el estado civil casada $(p<0,05)$, (Tabla 2) asimismo con el valor OR: 2,612 (IC: 1,156-5,904) Se encontró que la satisfacción en las madres primerizas es del $19,04 \%$ y el porcentaje de satisfacción de las que tienen más de un hijo es del $80,06 \%$. Se puede afirmar que hay relación significativa $(p<0,05)$ y con los valores de OR: 0,353 $(<1)$ y el IC: $0,140-0,82$

Ninguna dimensión supera el $40 \%$ de satisfacción global, siendo la dimensión seguridad la que más nivel de satisfacción presentó, (Gráfico 1) y las dimensiones de aspectos tangibles y capacidad de respuesta son las que presentan mayor grado de insatisfacción.

El $94,1 \%$ indican insatisfacción en la dimensión aspectos tangibles: existe ausencia de carteles de orientación, falta de comodidad en sala de espera, falta de equipos para la atención. El 91,2\% está insatisfecho en la dimensión capacidad de respuesta ya que indican mucho tiempo de espera para laboratorio, farmacia, rayos $\mathrm{x} y$ para la atención en el consultorio. El 85,3\% refiere insatisfacción respecto a la dimensión fiabilidad: citas no programadas 0 mal programadas, no encontrarse la historia clínica lista para la atención, y falta de orientación al público. El $78,4 \%$ está insatisfecho en la dimensión empatía: el médico no escucha atentamente, no muestra interés, no comprenden la explicación médica y el porqué de los análisis. El $77,5 \%$ se encuentran insatisfechos respecto a la seguridad ya que probablemente: no hay privacidad en la consulta, el médico no inspira confianza, no realizan exámen completo, y/o no despeja dudas de las madres. Se encuentra relación estadísticamente significativa $p<0,05$ con la 
satisfacción el grado de instrucción secundaria (Tabla 3), lo mismo sucede con el estado civil casado $(p=0,021)$; además al encontrarse el OR en 0,386 (IC: 0,151-0,990) para grado de instrucción secundaria y OR: 0,354 para el estado civil casado (IC: $0,147-$
0,857), en ambos casos menor a 1, podemos decir que no está asociado a una mayor ocurrencia de percepción de satisfacción si se encuentra en una madre dos o más características paralelamente.

Tabla 1. Satisfacción Global de madres de los recién nacidos Hospital III Yanahuara

\begin{tabular}{ccc}
\hline SATISFACCIÓN GLOBAL & $\mathbf{N}: \mathbf{1 0 2}$ & $\%: \mathbf{1 0 0}$ \\
\hline SATISFECHO & 42 & 41,20 \\
NO SATISFECHO & 60 & 58,80 \\
TOTAL & 102 & 100,0 \\
\hline Fuente: SERVQUAL & &
\end{tabular}

Tabla 2: Estado civil y numero de hijo asociado a la satisfacción de las madres de los recién nacidos que acuden a consultorio de Neonatología en el hospital III Yanahuara 2017.

\begin{tabular}{|c|c|c|c|c|c|}
\hline \multirow{3}{*}{ CARACTERÍSTICA } & \multicolumn{4}{|c|}{ SATISFACCIÓN } & \multirow{3}{*}{$\begin{array}{l}X^{2} \\
P \\
\text { OR } \\
\text { IC }\end{array}$} \\
\hline & \multicolumn{2}{|c|}{ SI } & \multicolumn{2}{|c|}{ NO } & \\
\hline & № & $\%$ & № & $\%$ & \\
\hline ESTADO CIVIL & & & & & $\begin{array}{l}X^{2}: 5,44 \\
P: 0,020\end{array}$ \\
\hline CASADA & 23 & 54,76 & 19 & 45,24 & OR:2,612 \\
\hline SOLTERA & 19 & 31,73 & 41 & 68,27 & IC: $1,156-5,904$ \\
\hline $\begin{array}{c}\text { NÚMERO DE HIJO } \\
1 \text { HIJO } \\
\text { MÁS DE } 1 \text { HIJO }\end{array}$ & $\begin{array}{c}8 \\
34\end{array}$ & $\begin{array}{l}19,04 \\
80,06\end{array}$ & $\begin{array}{l}24 \\
36\end{array}$ & $\begin{array}{l}40,03 \\
59,97\end{array}$ & $\begin{array}{c}\text { X2: } 4,845 \\
\text { p: } 0,028 \\
\text { OR: } 0,353 \\
\text { IC : } 0,140-0,82\end{array}$ \\
\hline
\end{tabular}

Gráfico 1. Nivel de Satisfacción según dimensiones de las madres de los recién nacidos que acuden a consultorio de Neonatología en el Hospital III Yanahuara 2017.

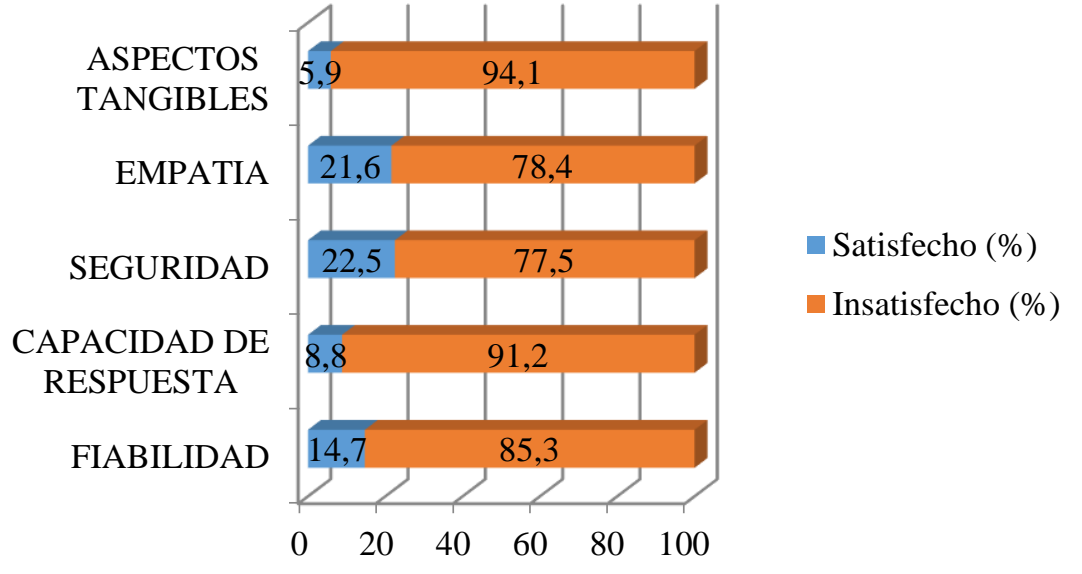

Fuente: SERVQUAL 
Tabla 3. Análisis Multivariado

\begin{tabular}{lllcc}
\hline & CALIDAD & $\mathrm{p}$ & $\mathrm{OR}$ & IC \\
\hline GRADO DE INSTRUCCIÓN & 3,923 & $\mathbf{0 , 0 4 8}$ & $\mathbf{0 , 3 8 6}$ & $0,151-0,990$ \\
LUGAR DE RESIDENCIA & $\mathbf{1 , 8 4 4}$ & 0,175 & 0,450 & $0,142-1,425$ \\
NÚMERO DE HIJO & 3,562 & $\mathbf{0 , 0 5 9}$ & 0,382 & $0,140-1,038$ \\
TIPO PARTO & 0,0700 & 0,791 & 1,137 & $0,438-2,953$ \\
ESTADO CIVIL & 5,298 & $\mathbf{0 , 0 2 1}$ & $\mathbf{0 , 3 5 4}$ & $0,147-0,857$ \\
\hline
\end{tabular}

Fuente: SERVQUAL

\section{DISCUSIÓN:}

No existen instrumentos específicamente validados para estudiar la satisfacción de los padres con la atención del control del niño sano. Aunque se han realizado estudios sobre la satisfacción del paciente adulto del consultorio externo empleando instrumentos como el SERVQUAL, este tipo de instrumentos no incluyen algunas dimensiones recomendadas para valorar la satisfacción en esta situación específica(8). Para garantizar la sensación de intimidad, la persona que las realizaba no había participado en el proceso asistencial y explicaba en cada caso que la información aportada no sería utilizada nunca para identificar a quien respondía. Por todo ello consideramos que las opiniones obtenidas representan adecuadamente las percepciones de las madres de los recién nacidos. En el estudio de Herrero et al (8), encontramos similitudes con el rango de las edades maternas: 20-43 años, la media de edad de las madres hallada fue 31,9 años. El alto porcentaje de usuarias con educación superior y secundaria completa, indicaría probablemente mayor capacidad de comprensión a la encuesta aplicada pero a la vez mayor exigencia de la valoración de las expectativas y percepciones que tienen sobre el servicio de salud. Se puede observar que las solteras son un $59 \%$ de la población. Esto contrasta con el estudio de Herrero et al (8), en el que el $66,52 \%$ eran madres casadas. En la Encuesta Nacional Demográfica y de Salud 2015 (ENDES), presentado por el Instituto Nacional de Estadística e Informática (INEI), el 31,6\% de partos en el Perú se realizan mediante cesárea y, en los últimos cinco años, este tipo de procedimiento se incrementó en 11,2 puntos porcentuales, lo que corresponde con lo encontrado. En cuanto a las variables, afirmamos que habrá una mayor ocurrencia de percepción de satisfacción que si la madre es soltera; un estudio australiano (9), encuestó vía correo a las madres a los 6 meses de haber tenido un hijo; evaluó su opinión sobre los cuidados neonatales y comparó el grupo de las que tenían muy buena opinión frente al resto, sin encontrar diferencias según edad, nivel educativo o paridad, aunque sí encontraron menores puntuaciones en mujeres sin pareja, con menos ingresos y en las menores de 35 años. Se puede explicar porque dentro de los principales predictores de estrés para las madres, están el tener menores ingresos, y el ser pareja "de hecho" (2) , por lo que las madres solteras presumiblemente estarán angustiadas, así, sus expectativas podrían ser más altas y su percepción sobre la atención del recién nacido serían más severas. Podemos decir que está relacionado a una menor ocurrencia de percibir la atención médica como satisfactoria si la madre es primeriza, esto se puede explicar porque en la satisfacción del usuario intervienen dos factores: la expectativa y la experiencia (pues es a partir de esta última que se forman las expectativas); en ambos factores influyen los valores que tenga la 
madre y si este es su primer hijo las expectativas respecto de la atención que recibirá serían mayores. Respecto a esto, los resultados se parecen a los del trabajo realizado por Ramírez (2), quien pudo observar significancia respecto a la insatisfacción, especialmente en la demanda de más información en las madres primerizas de bebes hospitalizados. Algún autor ha probado con éxito que una intervención simple, como facilitar que tomen notas, puede mejorar el seguimiento de las recomendaciones del control del recién nacido en primerizas, interviniendo así en el aumento del porcentaje de satisfacción en esta población (10). Estos hallazgos armonizan con lo encontrado en otros estudios, fundamentalmente en relación con el antecedente de hijos previos y tratamiento de fertilidad pues, por ejemplo, McCormick (12), sí encontró que estos podían ser predictores de los niveles de satisfacción (13). Se aprecia que existe una insatisfacción global en un $58,8 \%$, lo que quiere decir que la madre percibe la atención menos de lo que esperaba, el instrumento utilizado se ocupa de evaluar aspectos varios. En EsSALUD un estudio de percepción del usuario externo de la calidad evaluando las 5 dimensiones de la calidad realizado por Estupiñan (14), en el año 2010, de 5 hospitales de la Red Asistencial Rebagliati obtuvo que el $74 \%$ percibió que la calidad del servicio fue buena, el $19 \%$ regular y el $7 \%$ mala. Siendo el Hospital de Cañete (87\%) donde se percibió una mejor calidad de servicio, seguido de los Hospitales Angamos (84\%), Uldarico Roca (82\%), Hospital Rebagliati (74\%) y Alcántara $(50 \%)$. Donde se puede observar que la percepción promedio entre los hospitales de EsSALUD donde se ha realizado este tipo de encuesta es del $65 \%$ de satisfacción. Se observó que la satisfacción global de las madres del estudio se encuentra en un estándar menor de $60 \% \quad(41,18 \%)$ comparado con otros estudios realizados en otros establecimientos de salud en los servicios de consultorio externo con la encuesta SERVQUAL, como el estudio de Casalino (7), en el 2008 que obtuvo una satisfacción global del $44,36 \%$, y el estudio de Ninamango (15), del 2014 con una satisfacción del 26,1\%. Así, se puede observar que cada establecimiento tiene su propia realidad, dependiendo de los factores sociodemográficos, servicio donde se realiza el estudio, nivel de complejidad de cada establecimiento, según estos los usuarios tiene sus propias expectativas y percepciones.

La importancia dada a la comunicación es concordante con la referida en otros estudios. En general, la adecuada comunicación entre los profesionales sanitarios y los pacientes son factores muy importantes para la satisfacción en todos los ámbitos de la atención sanitaria y en diferentes países (16). Los padres quieren que la información médica les sea comunicado de manera que puedan comprenderla y en el momento más adecuado (17), tanto por parte de los médicos como de las enfermeras. Obviamente, la forma en que son informados influye en su nivel de satisfacción con la atención recibida (17). Es por eso que es importante que el personal sanitario esté formado en técnicas de comunicación.

Las madres expresaron que les hubiera gustado entender mejor sobre los cuidados y el tratamiento de su hijo. Este resultado, digno de ser tenido en cuenta, es un hallazgo similar al encontrado en otros estudios que muestran como los pacientes quieren participar más en la toma de decisiones sobre su tratamiento (18). En estos casos se da la circunstancia de que los pacientes pueden expresar que han podido comunicar a su médico sus puntos de vista sobre el tratamiento, mientras que al mismo tiempo pueden sentir que no tienen suficiente participación. Esta es una cuestión de difícil solución y se ha apuntado que un posible enfoque puede ser conseguir que las explicaciones de los médicos sean más fáciles de entender, para que las madres 
sientan que participan más en las decisiones sobre su tratamiento (19).

La intimidad está claramente relacionada con la satisfacción del paciente y algunos estudios señalan que los profesionales sanitarios sacrifican aspectos relacionados con la intimidad frente a cuestiones más técnicas (20). Es necesario incidir en la importancia de hacer siempre un esfuerzo para respetar al máximo este derecho de los pacientes

En el estudio de Estupiñan (14), la insatisfacción de la dimensión capacidad de respuesta fue de $43 \%$, en el Hospital Alcántara. El trato que reciben los pacientes y la percepción que estos tienen es muy distinto de hospital a hospital, en nuestro estudio la dimensión seguridad tuvo un nivel de insatisfacción de $77,50 \%$.

\section{CONCLUSIONES}

Existe un porcentaje de satisfacción del $58,80 \%$ respecto de la atención médica recibida por las madres que acuden al consultorio de neonatología del Hospital III Yanahuara. Entre las características sociodemográficas que se asocian con la satisfacción tenemos tener el estado civil casada, y que la madre tenga más de un hijo.

\section{RECOMENDACIONES}

Se recomienda sociabilizar el estudio a las autoridades del Hospital III Yanahuara para que se tomen las políticas y medidas necesarias para mejorar el establecimiento de salud, involucrando la mejora de la imagen Institucional, obteniendo un usuario satisfecho con atención oportuna, así mismo que las madres de todos los bebes se vayan con la cita tramitada al momento del alta de la madre o del bebe sí estuvo hospitalizado, así mismo se puede adicionar sillas y muebles en la sala de espera del consultorio externo para que las madres aguarden con mayor comodidad.

Se debe capacitar al personal para la orientación al usuario para corregir el mal trato que es percibido, además de una formación específica de los profesionales del servicio de neonatología respecto a la comunicación para mejorar la información y educación a los padres. Una información efectiva depende colectivamente de los padres, el personal sanitario y el sistema de salud. Se debe cuidar la comunicación con la familia, de forma que se realice con sensibilidad, respetando la dignidad e integridad del neonato y su familia y teniendo especial cuidado en que sea adecuada al nivel de conocimientos de cada entorno familiar

Es deber el colocar los letreros y carteles pertinentes para ubicar el consultorio de neonatología en el establecimiento. Se deben realizar trabajos de investigación del Servicio de Calidad de Salud con el usuario interno, para ver las causas del trato inadecuado al usuario externo para mejorar la dimensión de empatía.

\section{Correspondencia:}

MC. Karen Leticia Llerena Ururi

Correo electrónico:

Leticia.llerenau@hotmail.com

\section{REFERENCIAS BIBLIOGRÁFICAS}

1. Williams B. Patient satisfaction: a valid concept Soc Sci Med. 1994; 38(4):509- 16.

2. Ramírez Cuentas J. Estudio De Satisfacción De Los Padres En Una Unidad De Neonatología (Internet) España; 2011. (Citado el 14 de Enero del 2017) Disponible en: https://repositorio.unican.es/xmlui/bitstream /handle/10902/9628/Tesis\%20JHRC.pdf?s equence $=1$

3. Azoulay E, Pochard F, Chevret S, Lemaire $\mathbf{F}$, et al. Meeting the needs of Intensive Care Unit patient families: a multi-center study. Am J Respir Crit Care Med 2001; 163(1):135-9.

4. Institute of Medicine. Crossing the quality chasm: a new health system for the 21st century. Washington DC: Institute of Medicine; 2001. 
5. Asubonteg $\mathbf{P}$, Mc Cleary K, Swan J. SERVQUAL revisited: a critical review of service quality. J Serv Market 1996;(10) :62-81.

6. Cabello E. Validación Y Aplicabilidad De Encuestas SERVQUAL Modificadas para Medir la Satisfacción De Usuarios Externos En Servicios De Salud. Lima, Perú. Rev. Médica Heredia.2012; 23(2):88-95.

7. Casalino.E. Calidad de Servicio de la Consulta Externa de Medicina Interna de un Hospital General De Lima Mediante La Encuesta Servqual. Rev Soc Perú Med Interna 2008; 21.

8. Herrero J, Huidobro B, Amigo MC, Quiroga R, Fernández N. Valoración de las madres acerca de las recomendaciones para el cuidado del recién nacido al momento del alta hospitalaria. Buenos Aires, Argentina. Arch Argent Pediatr 2015; 113(1):28-35

9. Brown SJ, Davey MA, Bruinsma FJ. Women's views and experiences of postnatal hospital care in the Victorian Survey of Recent Mothers 2000. Midwifery 2005; 21(2):109-26. 13.

10. Berthod $\mathbf{P}$. Les enfants nés avant terme: la couveuse et la gavage à maternité.Paris; 1887.

11. Kistin CJ, Barrero-Castillero A, Lewis S, Hoch R, et al. Maternal note-taking and infant care: a pilot randomized controlled trial. Arch Dis Child 2012; 97(10):916-8.

12. McCormick MC, Escobar GJ, Zheng Z, Richardson DK. Factors influencing parental satisfaction with neonatal intensive care among the families of moderately premature infants. Pediatrics. 2008;121(6):1111-8.

13. Kenagy J, Berwick D, Shore M. Service quality in health care. JAMA 1999; 281: 661-5.

14. Estupiñan M. Percepción del usuario externo de la calidad de servicio en hospitalización pediátrica de la Red
Asistencial Rebagliati - Essalud. Lima, Perú. Rev. de la Facultad de Medicina Humana de la Universidad Ricardo Palma 2012; (1): 29-36.

15. Ninamango W. Percepción de la calidad de servicio de los usuarios en el consultorio externo de medicina interna del Hospital Nacional Arzobispo Loayza en enero del 2014. Tesis para optar el título de médico cirujano. Lima, Perú.; Facultad de Medicina, Universidad Nacional Mayor de San Marcos; 2014.

16. $\mathrm{Ng} \mathrm{CW}$, Lim GH, McMaster F, Molina J, Seow E, Heng BH. Patient satisfaction in an observation unit: the Consumer Assessment of Health Providers and Systems Hospital Survey. Emerg Med J. 2009; 26(8):586-9.

17. Informes estudios e investigación .Ministerio de Sanidad Servicios Sociales e Igualdad 2014 .Unidades de Neonatología. Estándares y recomendaciones de calidad. Madrid; 2014.

18. Bridges J, Flatley M, Meyer J. Older people's and relatives' experiences in acute care settings: systematic review and synthesis of qualitative studies. Int $\mathrm{J}$ Nurs Stud. 2010; 47(1):89-107.

19. Reátegui L. Calidad del servicio de emergencia desde la percepción del usuario externo del Hospital Sergio Bernales Collique. Tesis de Maestría. Lima, Perú. Universidad Privada Cayetano Heredia; 2008.

20. Larsen DL, Attkisson CC, Hargreaves WA, Nguyen TD. Assessment of client/patient satisfaction: development of a general scale. Eval Program Plann. 1979; 2(3):197- 207.

Recibido: 14/11/17

Aprobado para Publicación: 13/03/18 